\title{
Observation of an excess of events in the Higgs boson search in ATLAS
}

\author{
Robert D. Harrington ${ }^{\mathrm{a}, *}$, On behalf of the ATLAS Collaboration \\ ${ }^{a}$ SUPA - School of Physics and Astronomy, University of Edinburgh, Edinburgh, United Kingdom
}

\begin{abstract}
A search for the Standard Model Higgs boson with the ATLAS experiment at the LHC is presented. The $p p$ collisions datasets correspond to an integrated luminosity of up to $4.9 \mathrm{fb}^{-1}$ collected at $\sqrt{s}=7 \mathrm{TeV}$ in 2011 , and up to $5.9 \mathrm{fb}^{-1}$ collected at $\sqrt{s}=8 \mathrm{TeV}$ in 2012. Searches for $H \rightarrow \gamma \gamma, H \rightarrow Z Z^{(*)}, H \rightarrow W W^{(*)}, H \rightarrow b \bar{b}$ and $H \rightarrow \tau^{+} \tau^{-}$have been performed on the 2011 data, while $H \rightarrow \gamma \gamma$ and $H \rightarrow Z Z^{(*)} \rightarrow 4 l$ have been updated using both 2011 and 2012 data. The Standard Model Higgs boson has been excluded at 95\% confidence level for masses in the range of 110 to 122.6 $\mathrm{GeV}$ and 129.7 to $558 \mathrm{GeV}$. An excess of events has been observed for a Higgs boson mass hypothesis near 126.5 $\mathrm{GeV}$ with a local significance of $5.0 \sigma$.
\end{abstract}

Keywords: LHC, ATLAS, Higgs, Observation

\section{Introduction}

The ATLAS detector [1] was built to observe a wide spectrum of events from proton-proton $(p p)$ collisions produced at the Large Hadron Collider (LHC). In the Standard Model (SM) [2-4], electroweak symmetry breaking is provided by the Higgs mechanism, resulting in a new particle referred to as the Higgs boson [5-10]. While the production cross section and branching ratios can be determined for a given Higgs boson mass, $m_{H}$, the actual value of the Higgs boson mass is not known and the search must be performed over a wide range of Higgs boson masses. The combined LEP limit [11] excludes a SM Higgs boson with a mass below $114.4 \mathrm{GeV}$ at $95 \%$ CL, so ATLAS searches are performed for Higgs boson masses over $100 \mathrm{GeV}$.

Searches for the SM Higgs boson are being performed by the ATLAS Collaboration in several search channels based on the decay modes of the Higgs boson, as well as decays of subsequent vector bosons and tau leptons. The search channels are further broken down according to lepton flavor, presence of additional jets, kinematic regions, and other factors to enhance the sensitivity. An overview of the search channels is given in Table 1.

For the analyses presented in this paper, the overall signal strength factor $\mu$, which acts as a scale factor on

\footnotetext{
${ }^{*}$ Speaker

Email address: robert.duane.harrington.jr@cern.ch (Robert D. Harrington)
}

Preprint submitted to Nuc. Phys. (Proc. Suppl.) the total rate of signal events, is determined for each Higgs boson mass hypothesis. The signal strength is defined such that $\mu=0$ corresponds to background only, and $\mu=1$ corresponds to the SM Higgs boson signal in addition to the background. The fitting procedure used for individual analyses and the combination [12$15]$ is based on the profile likelihood ratio test statistic $\lambda(\mu)$ [16]. The test statistic allows to extracts the signal strength from the full likelihood which includes systematic uncertainties and their correlations. Exclusion limits are based on the $C L_{s}$ prescription [17] such that a value of $\mu$ is regarded as excluded at the $95 \%$ confidence level (CL) when $C L_{s}$ is less than $5 \%$.

In 2011, the LHC delivered to the ATLAS detector an integrated luminosity of $5.6 \mathrm{fb}^{-1}$ of $p p$ collisions at a centre-of-mass energy of $7 \mathrm{TeV}$. Between 4.6 and 4.9 $\mathrm{fb}^{-1}$ (depending on the data quality requirements of the specific channels) were used for 2011 analyses. These analyses performed on 2011 data were combined to report the indication of an excess with a mass near 126.5 $\mathrm{GeV}$ with a local significance of 2.0 standard deviations $(\sigma)$ [12]. The global probability for the background to create an excess of this significance in the mass range of $110-600 \mathrm{GeV}$ was estimated to be $\sim 15 \%$. The mass ranges from 110.0 to $117.5,118.5$ to 122.5 , and 129 to $539 \mathrm{GeV}$ were excluded at $95 \% \mathrm{CL}$.

As of July 2012, the LHC has delivered to the ATLAS detector an integrated luminosity of $6.6 \mathrm{fb}^{-1}$ at a centre-of-mass energy of $8 \mathrm{TeV}$. The higher centre-ofmass energy gives greater sensitivity to Higgs analy- 
ses since in most cases signal production cross sections increase more with higher centre-of-mass energy than background production cross sections. In addition, the instantaneous luminosities were higher in 2012 than in 2011, with peak luminosities almost double those of 2011 for the same $50 \mathrm{~ns}$ bunch spacing. Higher integrated luminosities give greater sensitivity, but they come with larger numbers of $p p$ collisions per bunch crossing (pile-up). The $H \rightarrow \gamma \gamma$ was shown to be relatively insensitive to pile-up, while the $H \rightarrow Z Z^{(*)} \rightarrow 4 l$ analysis has been improved to increase pile-up robustness. These analyses have been updated with 5.8 and $5.9 \mathrm{fb}^{-1}$ of the $20128 \mathrm{TeV}$ data, respectively.

\section{2011 High Mass Higgs Boson Searches}

In 2011, searches were performed up to $600 \mathrm{GeV}$ in the following channels: $H \rightarrow W W \rightarrow$ lvqq, $H \rightarrow Z Z \rightarrow$ $l l v v$ and $H \rightarrow Z Z \rightarrow l l q q$. The combination [18] of all channels was expected to allow exclusion at a $95 \%$ confidence level (CL) from 120-560 GeV. The actual excluded range using 2011 data alone was 129.2 to 541 $\mathrm{GeV}$. The combined exclusion plot is shown in Figure 1 .

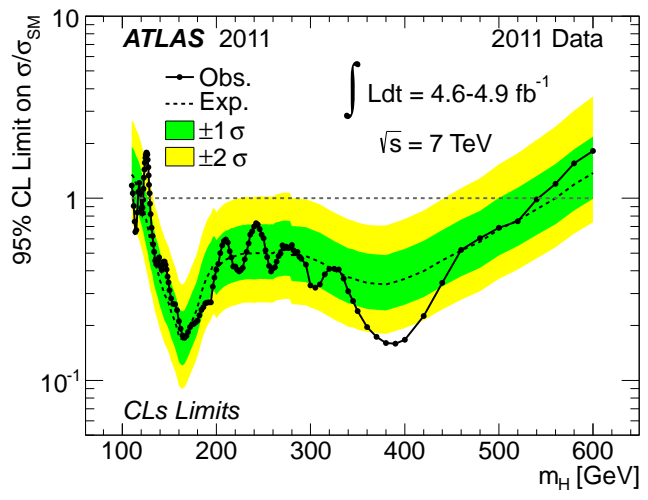

Figure 1: 2011 combination. The observed (full line) and expected (dashed line) $95 \%$ CL combined upper limits on the SM Higgs boson production cross section divided by the SM expectation as a function of $m_{H}$ in the full mass range considered. The dotted curves show the median expected limit in the absence of a signal and the green and yellow bands indicate the corresponding $68 \%$ and $95 \%$ intervals.[12]

\section{3. $2011 V H, H \rightarrow b \bar{b}$}

$H \rightarrow b \bar{b}[19]$ is the dominant decay mode in the low mass range, but backgrounds are prohibitively large for searches for Higgs bosons produced by gluon fusion, $g g \rightarrow H \rightarrow b \bar{b}$. To enhance the signal-background ratio, the $H \rightarrow b \bar{b}$ analysis was restricted to Higgs boson production in association with a vector boson, $W H$ or $Z H$, with the vector boson decaying to leptons. The analysis was performed in three channels based on the decay of the vector boson $(W \rightarrow l v, Z \rightarrow l l, Z \rightarrow v v)$. Analyses used the transverse momentum of the vector boson or $E_{T}^{m i s s}$ to enhance sensitivity. Fits to sidebands and control regions were used for background normalisations when possible, and likelihoods were formed from $m_{b b}$ distributions. 4.6 to $4.7 \mathrm{fb}^{-1}$ of 2011 data were analysed. No significant excess was found in the mass range 110-130 GeV (Figure 2), and an upper limit of 2.7 to 5.3 $\times \sigma_{S M}$ was set with $95 \%$ CL.

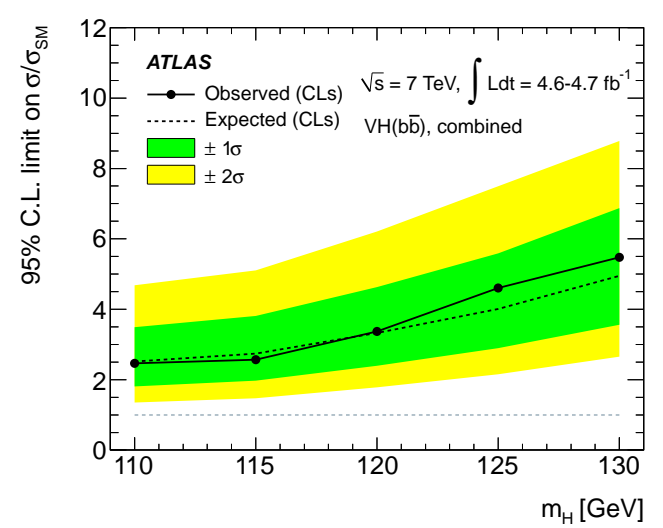

Figure 2: VH, $H \rightarrow b \bar{b}$. Expected (dashed) and observed (solid line) exclusion limits expressed as the ratio to the SM Higgs boson cross section, using the profile-likelihood method with CLs. The dark (green) and light (yellow) areas represent the 1 and 2 standard-deviation ranges of the expectation in the absence of a signal.[19]

\section{4. $2011 H \rightarrow \tau^{+} \tau^{-}$}

$H \rightarrow \tau^{+} \tau^{-}$[20] is a promising channel in spite of the low branching fraction. Three channels were analysed based on the decay of the $\tau$ leptons $\left(\tau_{\text {lep }} \tau_{\text {lep }}, \tau_{\text {lep }} \tau_{\text {had }}\right.$, $\left.\tau_{\text {had }} \tau_{\text {had }}\right)$, and these three channels were further subdivided with selections requiring jets to enhance the boost of the Higgs boson. Requiring a boosted Higgs boson aids in $\tau$ identification since the $\tau$ leptons are closer together, and $\tau$ decay products are more well collimated. $4.7 \mathrm{fb}^{-1}$ of 2011 data were analysed. As shown in Figure 3, no significant excess was observed, and an upper limit was placed on Higgs boson production of 3.2 to $7.9 \times \sigma_{S M}$ at $95 \% \mathrm{CL}$. 
Table 1: Summary of the individual channels used in the combination. The transition points between separately optimised $m_{H}$ regions are indicated when applicable. The symbols $\otimes$ and $\oplus$ represent direct products or sums over sets of selection requirements.

\begin{tabular}{|c|c|c|c|c|}
\hline Higgs Decay & $\begin{array}{l}\text { Subsequent } \\
\text { Decay }\end{array}$ & Sub-Channels & $m_{H}$ Range & $\mathcal{L}\left(\mathrm{fb}^{-1}\right)$ \\
\hline$V H \rightarrow b \bar{b}$ & $\begin{array}{l}Z \rightarrow v \bar{v} \\
W \rightarrow l v \\
Z \rightarrow l l\end{array}$ & $\begin{array}{l}E_{T}^{\text {miss }} \in\{120-160,160-200, \geq 200 \mathrm{GeV}\} \\
p_{T}^{W} \in\{<50,50-100,100-200, \geq 200 \mathrm{GeV}\} \\
p_{T}^{W} \in\{<50,50-100,100-200, \geq 200 \mathrm{GeV}\}\end{array}$ & $110-130$ & $\begin{array}{l}4.6 \\
4.7 \\
4.7 \\
\end{array}$ \\
\hline$H \rightarrow \tau^{+} \tau^{-}$ & $\begin{array}{l}\tau_{\text {lep }} \tau_{\text {lep }} \\
\tau_{\text {lep }} \tau_{\text {had }} \\
\tau_{\text {had }} \tau_{\text {had }}\end{array}$ & $\begin{array}{l}\{e u\} \otimes\{0 \text {-jets }\} \otimes\{l l\} \oplus\{1 \text {-jet,2-jets, } V H\} \\
\{e, \mu\} \otimes\{0 \text {-jets }\} \otimes\left\{E_{T}^{\text {miss }}<20, E_{T}^{\text {miss }} \geq 20 \mathrm{GeV}\right\} \oplus\{e, \mu\} \otimes \\
\{1 \text {-jet }\} \otimes\{l\} \otimes\{2 \text {-jets }\} \\
\{1 \text {-jet }\}\end{array}$ & $100-150$ & 4.7 \\
\hline$H \rightarrow W W^{(*)}$ & $\begin{array}{l}l v l v \\
l v q q^{\prime}\end{array}$ & $\begin{array}{l}\{e e, e \mu, \mu \mu\} \otimes\{0 \text {-jets, } 1 \text {-jet,2-jets }\} \otimes\{\text { low,high pile-up }\} \\
e, \mu \otimes\{0 \text {-jets, } 1 \text {-jet,2-jets }\}\end{array}$ & $\begin{array}{l}110-600 \\
300-600\end{array}$ & $\begin{array}{l}4.7 \\
4.7\end{array}$ \\
\hline$H \rightarrow \gamma \gamma$ & - & $\left\{p_{T_{t}} \otimes \eta_{\gamma} \otimes\right.$ conversion $\} \oplus\{\mathrm{VBF}\}$ & $110-150$ & $4.9+5.9$ \\
\hline$H \rightarrow Z Z^{(*)}$ & $\begin{array}{l}l l l l \\
l l v \bar{v} \\
l l q q\end{array}$ & $\begin{array}{l}\{4 e, 2 e 2 \mu, 2 \mu 2 e, 4 e\} \\
\{e e, \mu \mu\} \otimes\{\text { low,high pile-up }\} \\
\{b \text {-tagged, untagged }\}\end{array}$ & $\begin{array}{c}110-600 \\
200-280-600 \\
200-300-600\end{array}$ & $\begin{array}{l}4.8+5.8 \\
4.7 \\
4.7\end{array}$ \\
\hline
\end{tabular}

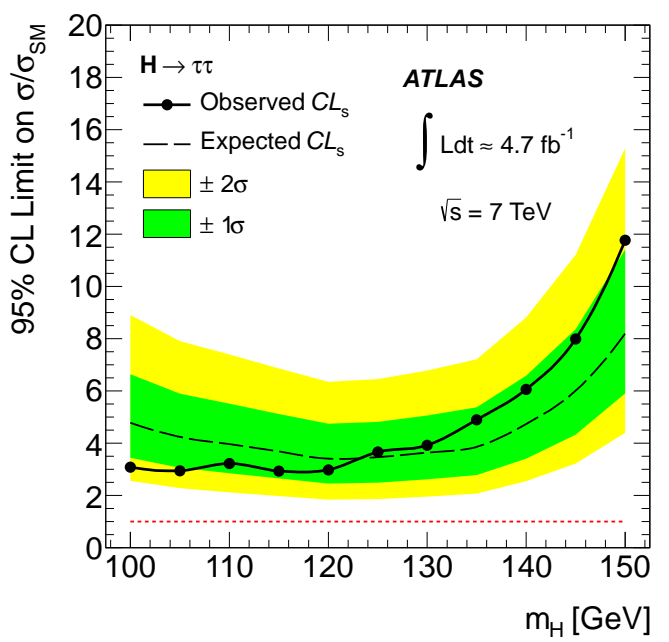

Figure 3: $H \rightarrow \tau^{+} \tau^{-}$. Expected (dashed) and observed (solid line) exclusion limits expressed as the ratio to the SM Higgs boson cross section, using the profile-likelihood method with CLs. The dark (green) and light (yellow) areas represent the 1 and 2 standard-deviation ranges of the expectation in the absence of a signal.[20]

\section{5. $2011 H \rightarrow W W^{(*)} \rightarrow l v l v$}

The $H \rightarrow W W^{(*)} \rightarrow l v l v$ [21] analysis is sensitive over the entire range of $110-600 \mathrm{GeV}$. For this channel, only $W$ bosons decaying to electrons and/or muons were considered. Backgrounds were normalised to data when possible. The Higgs boson transverse mass, calculated directly from lepton $p_{T}$ and $E_{T}^{\text {miss }}$, was used for the likelihood fit. To enhance sensitivity, events were separated into different categories in the likelihood fit based on the number of jets. $4.7 \mathrm{fb}^{-1}$ of 2011 data were analysed. As shown in Figure 4, no significant excess was observed. A SM Higgs boson with mass in the range of 133-261 $\mathrm{GeV}$ was excluded with $95 \% \mathrm{CL}$.

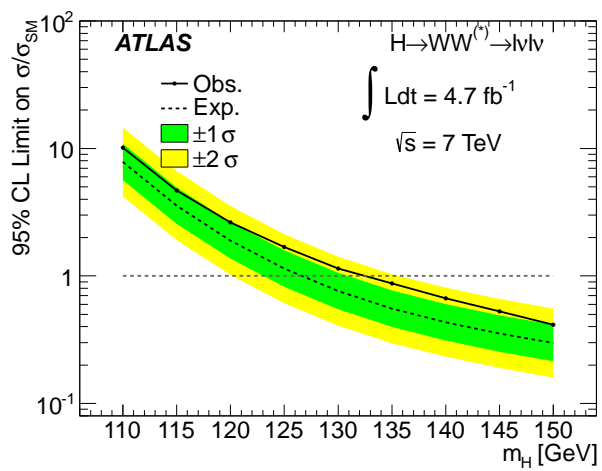

Figure 4: $H \rightarrow W W^{(*)} \rightarrow l v l v$. Expected (dashed) and observed (solid line) exclusion limits expressed as the ratio to the SM Higgs boson cross section, using the profile-likelihood method with CLs. The dark (green) and light (yellow) areas represent the 1 and $2 \sigma$ ranges of the expectation in the absence of a signal.[21]

\section{6. $2011+2012 H \rightarrow Z Z^{*} \rightarrow 4$ leptons}

The $H \rightarrow Z Z^{*} \rightarrow 4 l$ analysis [22] was updated with 2012 data for a total of $4.8 \mathrm{fb}^{-1}$ of $7 \mathrm{TeV}$ data and 5.8 $\mathrm{fb}^{-1}$ of $8 \mathrm{TeV}$ data. The likelihood was constructed using $m_{4 l}$ distributions for signals and backgrounds. Templates were taken from Monte Carlo simulation for the four signal contributions $(g g \rightarrow H$, VBF, $W H, Z H)$ and the $Z Z^{(*)}$ background. Shapes were taken from control regions in data for the $t \bar{t}, Z+$ jets and $Z b \bar{b}$ backgrounds and normalised to data. Four categories were used based 
on the invariant mass of the lepton pairs and their flavour (electrons or muons). Figure 5 shows the fit of the data to the $m_{4 l}$ templates for signal at 3 values of $m_{H}$ and backgrounds for all categories combined.

The analysis was improved over the 2011 analysis [23] through changes to selection cuts and electron reconstruction and identification to improve the sensitivity by $20-30 \%$. The resulting exclusion range was $131-$ 162 and $170-460 \mathrm{GeV}$ at $95 \% \mathrm{CL}$. An excess was observed at $125 \mathrm{GeV}$ with a local significance of $3.4 \sigma$, and a global significance of $2.5 \sigma$ taking into account the look-elsewhere effect over the range $110-141 \mathrm{GeV}$ (Figure 6). The fitted signal strength at $125 \mathrm{GeV}$ was $\mu=1.3 \pm 0.6$.

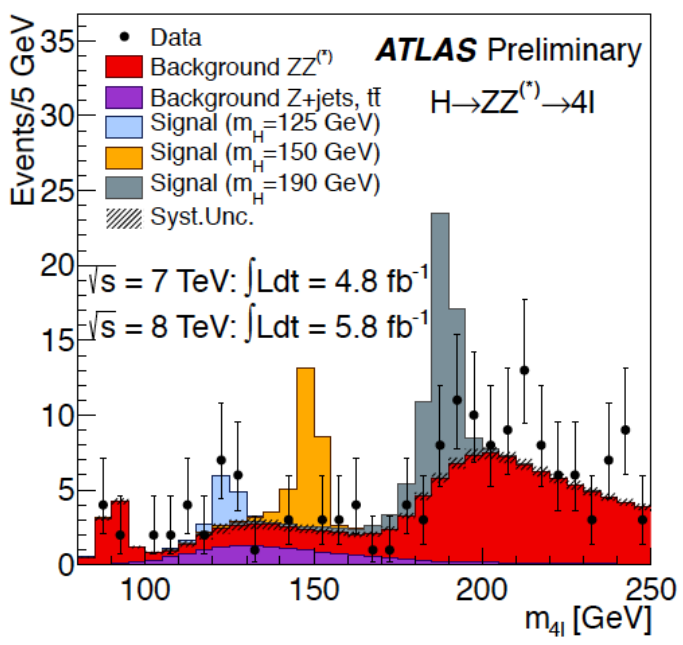

Figure 5: $H \rightarrow Z Z^{(*)} \rightarrow 4 l$. The distribution of the four-lepton invariant mass, $m_{4 l}$, for the selected candidates compared to the background expectation for the $80-250 \mathrm{GeV}$ mass range for the combined $\sqrt{s}=8 \mathrm{TeV}$ and $\sqrt{s}=7 \mathrm{TeV}$ datasets. Error bars represent $68.3 \%$ central confidence intervals. The signal expectation for several $m_{H}$ hypotheses is also shown.[22]

\section{7. $2011+2012 H \rightarrow \gamma \gamma$}

The $H \rightarrow \gamma \gamma$ search [24] was performed in the mass range 110 to $150 \mathrm{GeV}$ using integrated luminosities of $4.9 \mathrm{fb}^{-1}$ of $7 \mathrm{TeV} 2011$ data and $5.9 \mathrm{fb}^{-1}$ of $8 \mathrm{TeV}$ 2012 data. Nine categories were used in the likelihood based on the pseudorapidity of each photon, whether it was reconstructed as a converted or unconverted photon, and the di-photon momentum transverse to the diphoton thrust axis. A tenth category was used requiring 2 jets to enhance the contribution from vector boson fusion (VBF). The shape of the $m_{\gamma \gamma}$ distribution for the signal component of the likelihood was modelled with the sum

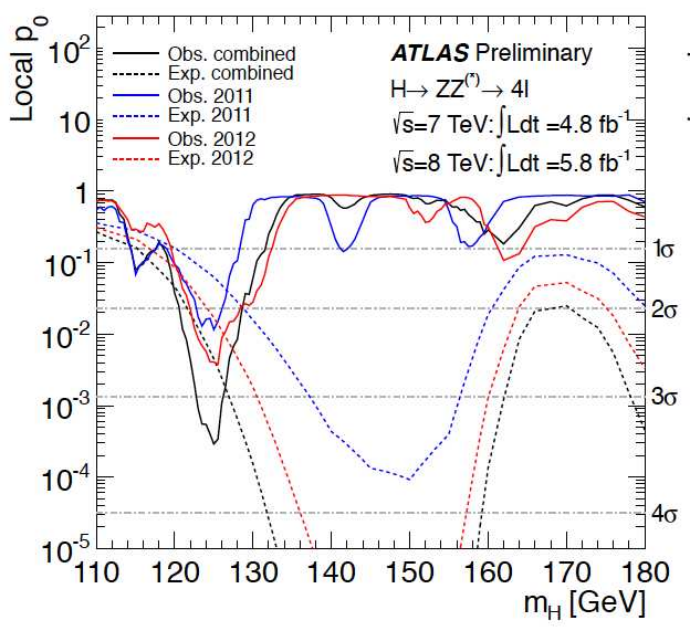

Figure 6: $H \rightarrow Z Z^{(*)} \rightarrow 4 l$. The observed local p0 for the combination of the 2011 and 2012 datasets (solid black line); the $\sqrt{s}=7 \mathrm{TeV}$ and $\sqrt{s}=8 \mathrm{TeV}$ data results are shown in solid lines (blue and red, respectively). The dashed curves show the expected median local p0 for the signal hypothesis when tested at the corresponding $m_{H}$. The horizontal dashed lines indicate the p0 values corresponding to local significances of $1 \sigma, 2 \sigma, 3 \sigma$ and $4 \sigma$. [22]

of a Crystal Ball and Gaussian fit to Monte Carlo simulation. The background likelihood shapes were datadriven and modelled, depending on the category, as an exponential function, a fourth-order polynomial or an exponential function of a second-order polynomial. Figure 7 shows the level of agreement between the data and the signal + background model for all categories combined. The ranges 112-122.5 and 132-143 GeV were excluded with $95 \% \mathrm{CL}$, and an excess was observed at $126.5 \mathrm{GeV}$ with a local significance of $4.5 \sigma$ (Figure 8) and a fitted signal strength of $\mu=1.9 \pm 0.5$. The global significance taking into account the look-elsewhere effect over the range $110-150 \mathrm{GeV}$ was $3.6 \sigma$.

\section{Combined Results}

The 2011 results for all channels, as well as the updated results using 2011 and 2012 data for $H \rightarrow Z Z^{(*)} \rightarrow 4 l$ and $H \rightarrow \gamma \gamma$, were combined [25] to give an exclusion in the ranges of 110 to $122.6 \mathrm{GeV}$ and 129.7 to $558 \mathrm{GeV}$ at $95 \% \mathrm{CL}$ (Figure 9). An excess was observed at 126.5 $\mathrm{GeV}$ with a local significance of $5.0 \sigma$ (Figure 10). The best-fit value of signal strength was $\mu=1.2 \pm 0.3$ (Figure 11). The global significance taking into account the look-elsewhere effect over the range of 110-600 (110150) was 4.1(4.3) $\sigma$. Lastly, the $H \rightarrow Z Z^{(*)} \rightarrow 4 l$ and $H \rightarrow \gamma \gamma$ channels were used to obtain the contour plots in Figure 12. 


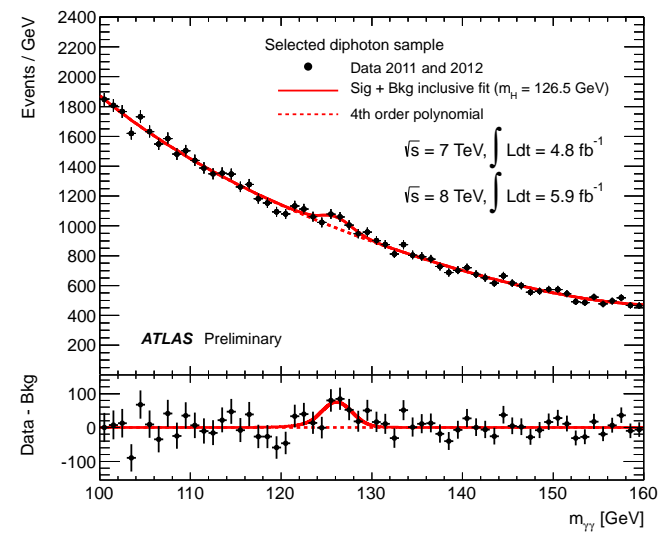

Figure 7: $H \rightarrow \gamma \gamma$. Invariant mass distribution for the combined $\sqrt{s}=7 \mathrm{TeV}$ and $\sqrt{s}=8 \mathrm{TeV}$ data samples. Superimposed is the result of a fit including a signal component fixed to a hypothesized mass of $126.5 \mathrm{GeV}$ and a background component described by a fourth-order Bernstein polynomial. The bottom inset displays the residual of the data with respect to the fitted background.[24]

\section{Conclusion}

Searches for the SM Higgs boson have been performed in the $H \rightarrow \gamma \gamma, H \rightarrow Z Z^{(*)}, H \rightarrow W W^{(*)}, H \rightarrow b \bar{b}$ and $H \rightarrow \tau^{+} \tau^{-}$channels with up to $4.9 \mathrm{fb}^{-1}$ of data taken in 2011 at $\sqrt{s}=7 \mathrm{TeV}$. The $H \rightarrow \gamma \gamma$ and $H \rightarrow Z Z^{(*)} \rightarrow 4 l$ analyses have been updated with up to $5.9 \mathrm{fb}^{-1}$ of data taken in 2012 at $\sqrt{s}=8 \mathrm{TeV}$. The new 2012 results were combined with the 2011 results to give an updated exclusion range. A $5 \sigma$ excess has been observed at a mass near $126.5 \mathrm{GeV}$, with an expected significance of $4.6 \sigma$. More data are needed to determine the nature of the newly observed resonance.

\section{References}

[1] ATLAS Collaboration, 2008 JINST 3 S08003.

[2] S. L. Glashow, Nucl. Phys. 22 (1961) 579588.

[3] S. Weinberg, Phys. Rev. Lett. 19 (1967) 12641266.

[4] A. Salam, in Elementary Particle Theory: Relativistic Groups and Analyticity (Nobel Symposium No. 8). (Almqvist and Wiksell, Stockholm, 1968) p. 367.

[5] F. Englert and R. Brout, Phys. Rev. Lett. 13 (1964) 321323

[6] P. W. Higgs, Phys. Lett. 12 (1964) 132133.

[7] P. W. Higgs, Phys. Rev. Lett. 13 (1964) 508509

[8] G. Guralnik, C. Hagen, and T. Kibble, Phys. Rev. Lett. 13 (1964) 585587.

[9] P. W. Higgs, Phys. Rev. 145 (1966) 11561163.

[10] T. Kibble, Phys. Rev. 155 (1967) 15541561.

[11] ALEPH, DELPHI, L3 and OPAL collaborations and LEP Working Group for Higgs boson searches, Phys. Lett. B 565 (2003) 6175.

[12] ATLAS Collaboration, Phys. Rev. D 86, 032003 (2012).

[13] ATLAS Collaboration, Eur. Phys. J. C71 (2010) 1728.

[14] ATLAS and CMS Collaborations, LHC Higgs Combination Working Group Report, ATL-PHYS-PUB-2011-011, CERNCMS-NOTE-2011-005 (2011).

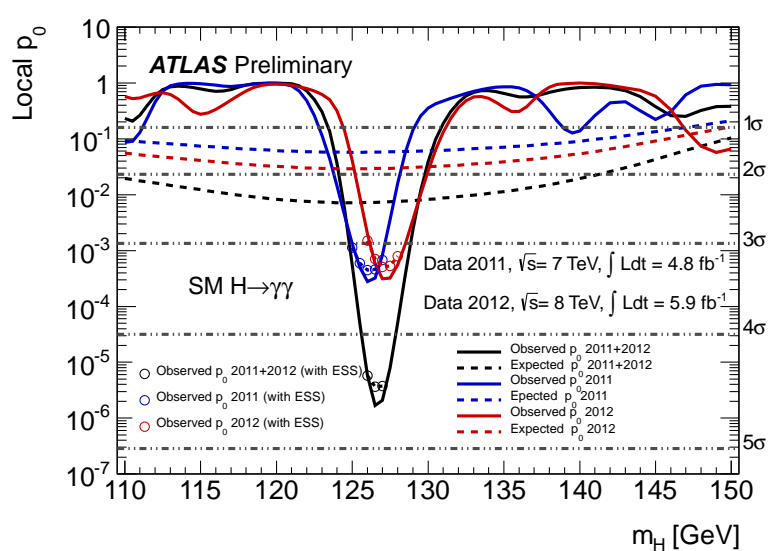

Figure 8: $H \rightarrow \gamma \gamma$. Expected and observed local p0 values for a SM Higgs boson as a function of the hypothesized Higgs boson mass $\mathrm{mH}$ for the combined analysis and for the $\sqrt{s}=7 \mathrm{TeV}$ and $\sqrt{s}=8 \mathrm{TeV}$ data samples separately. The observed $\mathrm{p} 0$ including the effect of the photon energy scale uncertainty on the mass position is included via pseudo-experiments and shown as open circles.[24]

[15] L.Moneta, K.Belasco, K.S.Cranmer, S.Kreiss, A.Lazzaro, et al., PoS ACAT2010 (2010) 057

[16] G.Cowan, K.Cranmer, E.Gross and O.Vitells, Eur. Phys. J. C71 (2010) 1554.

[17] A.L.Read, J.Phys G28 (2002) 2693-2704.

[18] ATLAS Collaboration, An update to the combined search for the Standard Model Higgs boson with the ATLAS detector at the LHC using up to $4.9 \mathrm{fb}^{1}$ of pp collision data at $\sqrt{s}=7 \mathrm{TeV}$, ATLAS-CONF-2012-019 (2012).

[19] ATLAS Collaboration, Search for the Standard Model Higgs boson produced in association with a vector boson and decaying to a b-quark pair with the ATLAS detector at the LHC, submitted to Phys. Lett. B (2012), arXiv:1207.0210 [hep-ex].

[20] ATLAS Collaboration, Search for the Standard Model Higgs boson in the $H \rightarrow \tau^{+} \tau^{-}$decay mode in $\sqrt{s}=7$ Tev pp collisions with ATLAS, JHEP 09 (2012) 070.

[21] ATLAS Collaboration, Search for the Standard Model Higgs boson in the $H \rightarrow W W^{(*)} \rightarrow W^{+} W^{-}$decay mode with $4.7 \mathrm{fb}^{-1}$ of ATLAS data at $\sqrt{s}=7 \mathrm{TeV}$, Phys.Lett. B 716 (2012) 62-81.

[22] ATLAS Collaboration, Observation of an excess of events in the search for the Standard Model Higgs boson in the $H \rightarrow$ $\mathrm{ZZ}^{(*)} \rightarrow 4$ l channel with the ATLAS detector, ATLAS-CONF2012-092 (2012)

[23] ATLAS Collaboration, Search for the Standard Model Higgs boson in the decay channel $H \rightarrow Z Z^{(*)} \rightarrow 4 l$ with $4.8 \mathrm{fb}^{-1}$ of pp collisions at $\sqrt{s}=7 \mathrm{TeV}$ with the ATLAS detector, Phys. Lett. B 710 (2012) 382-402.

[24] ATLAS Collaboration, Observation of an excess of events in the search for the Standard Model Higgs boson in the $\gamma \gamma$ channel with the ATLAS detector, ATLAS-CONF-2012-091 (2012).

[25] ATLAS Collaboration, Observation of an Excess of Events in the Search for the Standard Model Higgs boson with the ATLAS detector at the LHC, ATLAS-CONF-2012-093 (2012). 


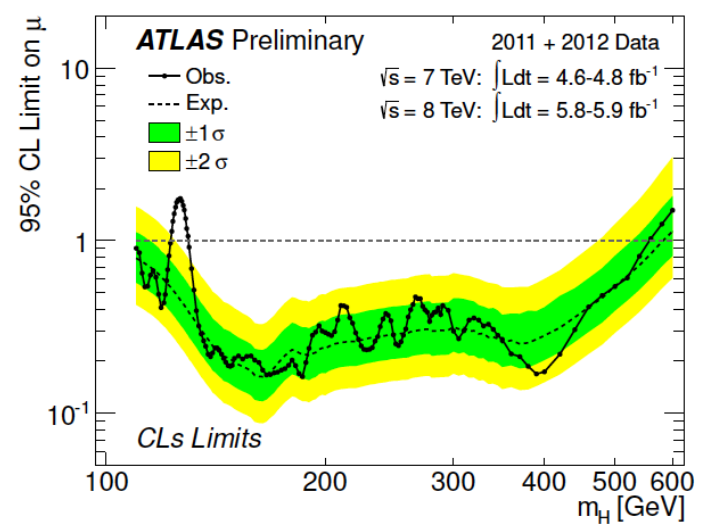

Figure 9: 2011+2012 combined. The observed (full line) and expected (dashed line) $95 \%$ CL combined upper limits on the SM Higgs boson production cross section divided by the SM expectation as a function of $m_{H}$ in the full mass range. The dashed curves show the median expected limit in the absence of a signal and the green and yellow bands indicate the 68\% and 95\% intervals.[25]

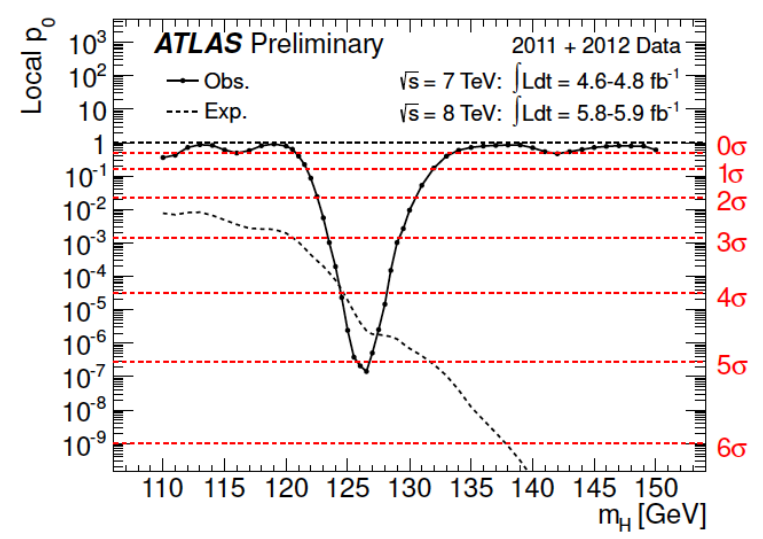

Figure 10: 2011+2012 combined. The local probability $p_{0}$ for a background-only experiment to be more signal-like than the observation in the low mass range as a function of $m_{H}$. The dashed curves show the median expected local $p_{0}$ under the hypothesis of a SM Higgs boson production signal at that mass. The horizontal dashed lines indicate the p-values corresponding to significances of $1 \sigma$ to $6 \sigma$.[25]

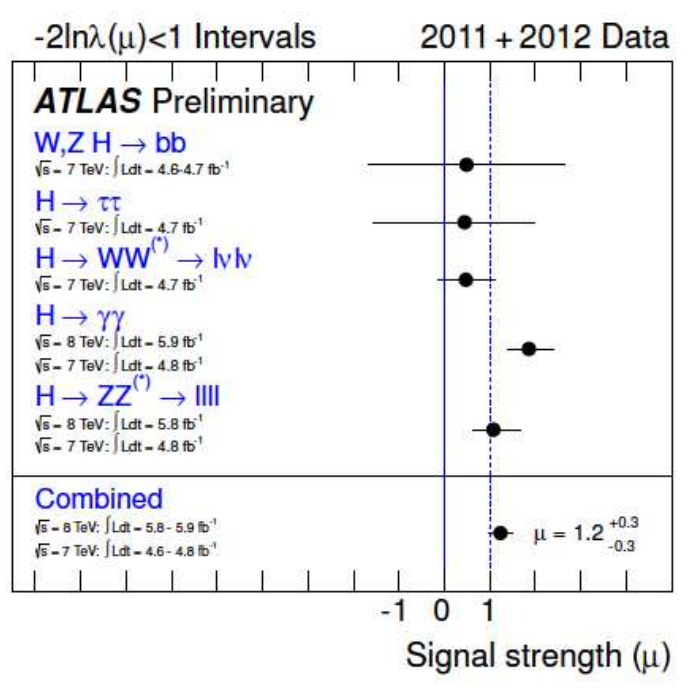

Figure 11: Summary of the individual and combined best-fit values of the strength parameter for a Higgs boson mass hypothesis of $126.5 \mathrm{GeV} .[25]$

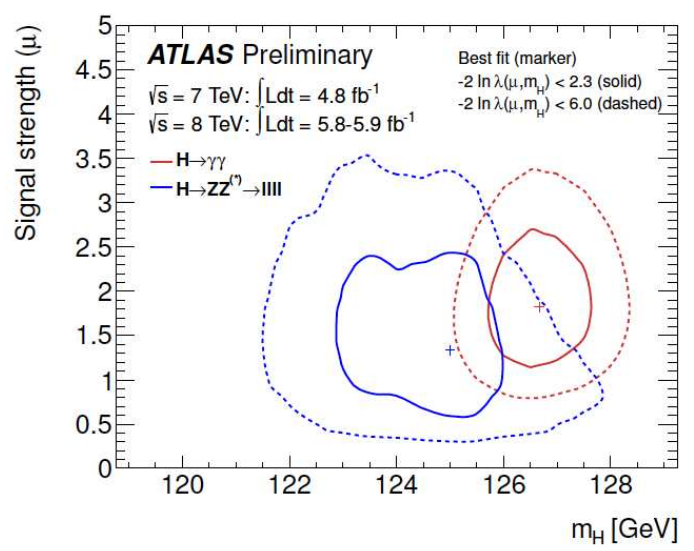

Figure 12: 2011+2012 combined. Likelihood contours in $\left(\mu, m_{H}\right)$ for the $H \rightarrow Z Z^{(*)} \rightarrow l^{+} l^{-} l^{+} l^{-}$and $H \rightarrow \gamma \gamma$ channels including energy scale systematics are shown in panel.[25] 